\title{
Proposed use of shared decision making (SDM) to facilitate informed patient choice in the management of cholestasis of pregnancy (ICP)
}

\begin{abstract}
Despite a paucity of data that intrahepatic cholestasis of pregnancy (ICP) is a risk for unexplained term fetal demise, active management to achieve delivery between $360 / 7$ and 37 0/7 weeks remains standard clinical practice. This practice continues despite conflicting evidence associating ICP with increased stillbirth risk, disregarding the simultaneous presence of voluminous data attesting to the perinatal and pediatric hazards associated with late preterm and early-term deliveries. This clinical risk correlation between possible antenatal death versus the potential long-term consequences for iatrogenic late preterm or early term infant born from an ICP affected pregnancy warrants inclusion of shared decision making to elicit the patient's values and preferences during the ICP management option informed consent process.
\end{abstract}

Keywords: bile acid, early term birth, full-term birth, intrahepatic cholestasis of pregnancy, patient decision aid, shared decision making
Volume II Issue 2 - 2020

\author{
Cassandra E Henderson,' Shadi Rezai, ${ }^{2}$ \\ Richard A Giovane ${ }^{3}$ \\ 'Garden OB/GYN, Mercy Medical Center, USA \\ ${ }^{2}$ Valley Community Healthcare, 680 I Coldwater Canyon \\ Avenue, USA \\ ${ }^{3}$ Regional Medical Center of Central Alabama, USA
} Correspondence: Cassandra E. Henderson MD, Garden OB/
GYN, Mercy Medical Center, New York, NY, USA,

Email cehendersonmd@gmail.com

Received: March 02, 2020 | Published: April 15, 2020
Abbreviations: BA, bile acid; ETB, early term birth; FTB, full-term birth; ICP, intrahepatic cholestasis of pregnancy; SDM, shared decision making

\section{Introduction}

In 2014 our clinical team in the Bronx, New York, produced a description of the evolution of active management for pregnancies affected by intrahepatic cholestasis (ICP). ${ }^{1}$ We concluded that there was little evidence to support this accepted preemptive obstetric management. Recently, our conclusion was supported by a controlled meta-analysis published in Lancet by Ovadia and colleagues. ${ }^{2}$ Ovadia found no significant difference in the stillbirth rate between the control and study population. Specifically, stillbirths occurred in $45(0.83 \%)$ of the study population of 5557 women affected by ICP versus 519 $(0.32 \%)$ of the 163947 in the control population (Odds ratio $\{\mathrm{OR}\}$ $1.46\{95 \%$ CI $0.73-2.89\}$; R $=59.8 \%$. It was only through a subgroup analysis of the group with serum bile acids in excess of $100 \mu \mathrm{mol} / 1$ (hazard ratio [HR] 30-50 \{95\% CL 8.83-105, 30\} p0.0001 did the authors identified an increased fetal demise risk.

Ovadia and colleagues were not able to control for known comorbid stillbirth risks such as maternal diabetes, ${ }^{3}$ hypertension, ${ }^{4}$ advanced maternal age ${ }^{5}$ or maternal obesity. ${ }^{6,7}$ Notably, in an aggregate data meta-analysis the Ovadia et al reported that when compared to the control population, ICP affected pregnancies had a higher BMI and prevalence of pre-eclampsia and gestational diabetes. The authors were not able to control for the lack of or insufficient prenatal care that is a documented risk factor for stillbirth. ${ }^{8}$

Despite the paucity of substantiated evidence that intrahepatic cholestasis of pregnancy (ICP) is an independent risk for unexplained term fetal stillbirth, active management to achieve delivery between $360 / 7$ and $370 / 7$ weeks remains standard clinical practice. ${ }^{1,9}$ This practice continues based on conflicting evidence associating ICP with increased stillbirth risk, disregarding the simultaneous presence of voluminous data attesting to the perinatal and pediatric hazards associated with late preterm and early term deliveries. The Society for
Maternal Fetal Medicine (SMFM) supports antepartum testing and delivery between 37 and 38 weeks of gestation while acknowledging the absence of evidenced based recommendation for timing of delivery in ICP affected pregnancies. ${ }^{10}$

SDM is appropriate for management and therapeutic decisions that are not urgently time sensitive and for which there is no clear best evidence based clinical option. A shared decision model should be used during the informed consent process to identify, respect and incorporate the patient values; beliefs and preferences in the selection of expectant or active management of an ICP affected pregnancy. As obstetric providers we often are not aware of the late neonatal or pediatric outcomes that affect infants we deliver. It is therefore appropriate to involve pregnant women in an objective discussion of the potential short and long-term risks associated with iatrogenic late preterm and early term delivery resulting from clinical concerns about ICP associated stillbirth risk.

In contrast to the limited evidence suggesting ICP is an independent risk for stillbirths, there is abundant evidence that early term delivery is positively associated with neonatal morbidity 11.9 The ACOG Committee Opinion No 765 calls for "Avoidance of Nonmedically Indicated Early Term Deliveries [Early Term Birth (ETB)] and Associated Neonatal Morbidities" indicating the evidence of the short term and long-term consequences of being born before 39 weeks are robust and indisputable. ${ }^{11}$ When compared to infants born full term [Full-Term Birth (FTB)] after 39 weeks of gestation, infants born early term at 37 to 38 weeks have increased morbidity and higher healthcare costs. ${ }^{12-14} \mathrm{~A}$ retrospective analysis of population based data indicates late preterm or early term birth finding birth between 37 and 38 weeks of gestation increased the individual's risk for developing diabetes and obesity. ${ }^{15}$

ETB when compare to FTB is frequently associated with neonatal, pediatric and adolescent respiratory illness that often lasts well into adulthood. ${ }^{16}$ ETB has also been associated with increased prevalence of pediatric obstructive sleep apnea. ${ }^{17}$ Furthermore, Chan E et al described in a systematic review of 8 studies, that when compared 
to FTB, ETB is associated with lower cognitive abilities identified during childhood that persisted into adulthood (adjusted risk ratio 1.38 [95\% CI 1.06-1.79] and increased special education needs. ${ }^{18,19}$

It is likely that for some individuals the potential benefit of preventing an unlikely term fetal demise may not outweigh the real possibility of iatrogenic neonatal or pediatric harm. Although, it is fundamental to the practice of obstetrics to oversee the safe delivery of a healthy newborn infant, long-term pediatric and adolescent consequences of perinatal decisions have not traditionally been a measured obstetric outcome. For the obstetrician and patient, the demise of a viable fetus is undoubtedly a horrible event, but for the patient and her family the increased risk for an offspring being affected by neonatal or long term health consequences, may be deemed an equally or more devastating outcome.

\section{Proposed ICP management protocol}

Informed decision with use of Shared Decision Making (SDM) tool to select management option. SDM is an appropriate tool as this is not a time sensitive decision and there is no clear best evidence based clinical option.

\section{a. Confirm ICP diagnosis}

Elevated Serum Bile Acids: $>100 \mathrm{~mol} / \mathrm{L}$

Absence of co-morbid conditions (i.e. maternal diabetes or hypertension, advanced maternal age or morbid obesity (BMI $>50)$, no prenatal care) that would independently warrant obstetric intervention.

b. Confirm the patient wants to participate in SDM.

c. Select Decisional Aid to use during the informed decisionmaking process with the health care provider.

d. Suggested Non-Disease Specific SDM Aids.

Option Grid and Ottawa Personal Decision Guide are examples of non-disease specific SDM aids (Figure 1).

Ottawa Hospital Research Institute Patient Decision Aids is available on-line (Direct Link: https://decisionaid.ohri.ca/) as an interactive tool that facilitates the identification and discussion of the individual values to determine the patient's most important treatment benefit while avoiding the most undesired risk. Patients use stars and zeros to indicate how important is to them to choose or avoid aspects of each option.

e. Use a shared decision-making tool to present the risks and benefits of active and expectant management of ICP.

Active management: Goal: To avoid fetal demise by infant delivery between 36 and 37weeks of gestation.

Review and present supporting evidence for short-term and longterm risks to the off spring.

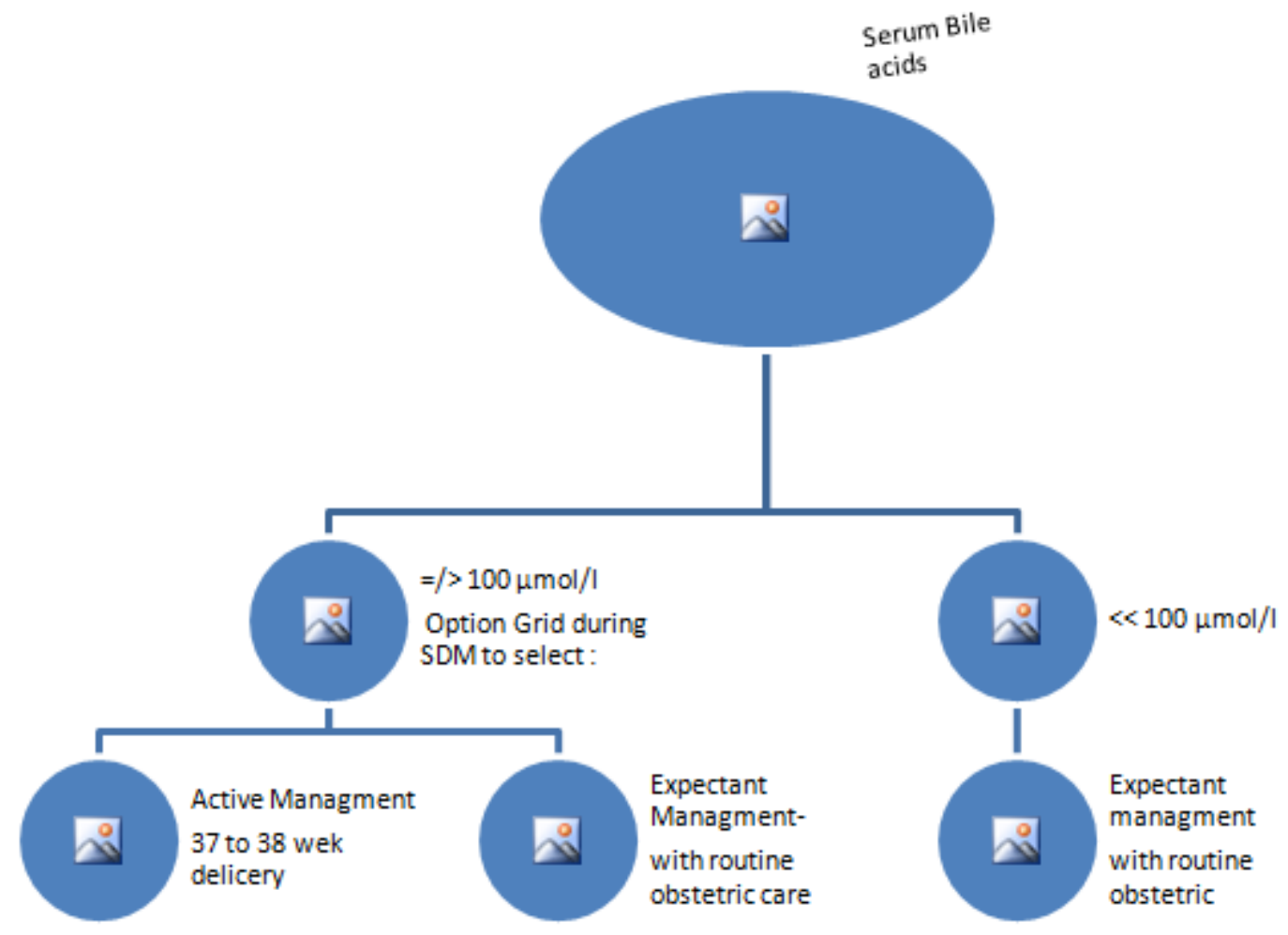

Figure I is a proposed ICP Option Grid for the ICP management options. For the offspring resulting from a pregnancy affected by ICP, the Option Grid presents their risk and benefits for each management option. The information provided is used to assist the patient in making an informed decision regarding active or expectant management of ICP. 
Expectant management: Goal: To continue prenatal care with management after 39 weeks by obstetric indications. To continuing serial fetal surveillance until delivery is prudent even in the absence of evidence to support this practice.

With use of shared decision- making tool, consideration may be given to elective induction of labor at 39 weeks of gestation. When compared to expectant management, elective induction at thirty-nine weeks is associated with a lower risk of cesarean delivery, maternal peripartum infection and adverse perinatal outcomes. ${ }^{20}$

\section{Summary}

Obstetric management decisions are grounded in avoiding preventable adverse perinatal outcome. However, as obstetric providers we are rarely involved and usually remain removed from the neonatal, pediatric or adolescent consequences resulting from our antenatal decision-making. The equipoise in perinatal outcome associated with active and expectant management of ICP affected pregnancies supports the use of SDM to address and acknowledge individual patient's values and preferences prior to choosing active or expectant ICP management (Table 1).

Table I ICP Management Option Grid; ICP is defined by the elevation of bile acids (BA). Earlier reports suggested elevated bile acids increased the risk a pregnancy would end with a stillbirth. Subsequent reports have only the risk of fetal death increases only if bile acids are more than $100 \mu m o l / L$. However, it is likely that any women with BA more than $100 \mu \mathrm{mol} / \mathrm{L}$ are not enrolled in prenatal care which is by itself a risk for stillbirth. Early delivery before 39 weeks will decrease the risk your baby will be stillborn. However, delivering your baby before 39 will also increase the risk your newborn infant will be admitted to the neonatal intensive care unit (NICU) for management of breathing illness, temperature and glucose management. In addition being born before 39 weeks is associated with an increased chance of long-term illness such as developmental delay, poor school performance and need for social security support services. ${ }^{21}$

Early or term deliver
What are bile acids (BA)
in ICP
in ICP

What is the risk my baby will die before it is born?

\section{Preterm delivery at 36 to 38 weeks}

a. Serum bile acids more than $40 \mu \mathrm{mol} / \mathrm{l}$

b. Serum bile acids more than 100 umol/l

a. $0.1 \%(3 / 2310)$ if $\mathrm{BA}>40 \mu \mathrm{mol} / \mathrm{L}$

b. $3.4 \%(18 / 524)$ if $\mathrm{BA}>100 \mu \mathrm{mol} / \mathrm{L}$

Continue pregnancy until labor occurs (expectant management)

Serum bile acids at normal range $0.0-11.3 \mathrm{mmol} / \mathrm{L}$

b. $\quad 1.0 \%(10.3 / 1000)$ for black women

c. $\quad 0.5 \%(5 / 1000)$ for Hispanic women

d. II.4\% (I.94 RR) if received no prenatal care $^{22}$

I.39 RR for respiratory disorders

What are the immediate effects on my baby

What are the long-term effects on my baby
Elective delivery after 39 weeks of gestation a. Breathing disorders during infancy

b. Increased risk for asthma or pneumonia as adolescent and young adult

Mental or learning challenges:

a. Between 36 and 39 weeks the IQ score decreases 1.94 points for each week $<39$ weeks of gestation

b. At 36weeks there is a I.38 RR increased need for special education for each week less than 39 weeks. ${ }^{23,24}$

c. Decreased brain development/lower grey matter volume when infants born at 36 weeks compared to those born at 39 weeks.
Less breathing disorders during infancy and adolescence

a. Improved learning ability, higher IQ test scores and less need for special education programs.

b. Need for special education 14/100 for births at 39 weeks versus $19 / 100$ for births at 36 weeks.
Lower risk of cesarean delivery, maternal peripartum infection and adverse perinatal outcomes. ${ }^{20}$ 


\section{Acknowledgments}

None.

\section{Funding}

None.

\section{Conflicts of interest}

The author and co-authors have no conflicts of interest relevant to this article.

\section{References}

1. Henderson CE, Shah RR, Gottimukkala S, et al. Primum non nocere: how active management became modus operandi for intrahepatic cholestasis of pregnancy. Am J Obstet Gynecol. 2014;211(3):189-196.

2. Ovadia C, Seed PT, Sklavounos A, et al. Association of adverse perinata outcomes of intrahepatic cholestasis of pregnancy with biochemical markers: results of aggregate and individual patient data meta-analyses. Lancet. 2019;393(10174):899-909.

3. Starikov R, Dudley D, Reddy UM. Stillbirth in the pregnancy complicated by diabetes. Curr Diab Rep. 2015;15(3):11.

4. Hirst JE, Villar J, Victora CG, et al. The antepartum stillbirth syndrome: risk factors and pregnancy conditions identified from the INTERGROWTH21st project. BJOG. 2018;125(9):1145-1153.

5. Waldenström U, Cnattingius S, Norman M, et al. Advanced maternal age and stillbirth risk in nulliparous and parous women. Obstet Gynecol. $2015 ; 126(2): 355-362$

6. Jacob L, Kostev K, Kalder M. Risk of stillbirth in pregnant women with obesity in the United Kingdom. Obes Res Clin Pract. 2016;10(5):574-579.

7. Yao R, Ananth CV, Park BY, et al. Obesity and the risk of stillbirth: a population-based cohort study. Am J Obstet Gynecol. 2014;210(5):457. e1-459.

8. KC Ashish, Nelin V, Wrammert J, et al. Risk factors for antepartum stillbirth: a case-control study in Nepal. BMC Pregnancy Childbirth. $2015 ; 15: 146$.

9. ACOG Committee Opinion No. 764: Medically indicated late-preterm and early-term deliveries. Obstet Gynecol. 2019;133(2):e151-e155.

10. SMFM Consult Series. Understanding intrahepatic cholestasis in pregnancy/ intrahepatic cholestasis of pregnancy explained. Contemporary $O B / G Y N .2011$
11. ACOG Committee Opinion No. 765: Avoidance of nonmedically indicated early-term deliveries and associated neonatal morbidities, Obstet Gynecol. 2019;133(2):e156-e163.

12. Helle E, Andersson S, Häkkinen U, et al. Morbidity and health care costs after early term birth. Paediatr Perinat Epidemiol. 2016;30(6):533-540.

13. Kotecha SJ, Watkins WJ, Lowe J, et al. Effect of early-term birth on respiratory symptoms and lung function in childhood and adolescence. Pediatr Pulmonol. 2016;51(11):1212-1221.

14. Harju M, Keski-Nisula L, Georgiadis L, et al, The burden of childhood asthma and late preterm and early term births, J Pediatr. 2014;164(2):295299.

15. Paz Levy D, Sheiner E, Wainstock T, et al. Evidence that children born at early term (37-38 6/7 weeks) are at increased risk for diabetes and obesityrelated disorders. Am J Obstet Gynecol. 2017;217(5):588.e1-588.e11.

16. Tita ATN, Jablonski KA, Bailit JL, et al. Neonatal outcomes of elective early-term births after demonstrated fetal lung maturity, Am J Obstet Gynecol. 2018; 219(3):296.e1-296.e8.

17. Walfisch A, Wainstock T, Beharier O, et al. Early term deliveries and the risk of pediatric obstructive sleep apnoea in the offspring. Paediatr Perinat Epidemiol. 2017;31(2):149-156.

18. Chan E, Leong P, Malouf R, et al. Long-term cognitive and school outcomes of late-preterm and early-term births: a systematic review. Child Care Health Dev. 2016;42(3):297-312.

19. MacKay DF, Smith GC, Dobbie R, et al. Gestational age at delivery and special educational need: retrospective cohort study of 407,503 schoolchildren. PLoS Med. 2010;7(6):e1000289.

20. Binarbaşı P, Akın Y, Narter F, et al. Mortality and morbidity in late-preterm newborns. Turk Pediatri Ars. 2013;48(1):17-22.

21. VanderWeele TJ, Lantos JD, Siddique J, et al. A comparison of four prenatal care indices in birth outcome models: comparable results for predicting small-for-gestational-age outcome but different results for preterm birth or infant mortality. J Clin Epidemiol. 2009;62(4):438-445.

22. Grobman WA, Caughey AB. Elective induction of labor at 39 weeks compared with expectant management: a meta-analysis of cohort studies. Am J Obstet Gynecol. 2019;221(4):304-310.

23. U.S. Department of Education, Office of Special Education Programs. Individuals with disabilities education act (IDEA) database. 2018.

24. National Center for Education Statistics. National elementary and secondary enrollment (NCES) projection model, 1972 through 2027. 